\title{
Lessons Learnt Implementing a Cardiovascular Disease Quality Improvement Intervention in Australian Primary Care: A Mixed Method Evaluation
}

\author{
Charlotte Hespe ( $\nabla$ charlotte.hespe@nd.edu.au ) \\ University of Note Dame Australia \\ Katrina Giskes \\ University of Note Dame Australia \\ Mark Harris \\ University of New South Wales \\ David Peiris \\ University of Sydney
}

\section{Research Article}

Keywords: Quality improvement, cardiovascular disease prevention, general practice, quality improvement collaboration

Posted Date: August 23rd, 2021

DOI: https://doi.org/10.21203/rs.3.rs-805739/v1

License: (a) (i) This work is licensed under a Creative Commons Attribution 4.0 International License. Read Full License 


\section{Abstract}

Background

There are discrepancies between evidence-based guidelines for screening and management of cardiovascular disease (CVD) and implementation in Australian general practice. Quality-improvement (QI) initiatives aim to reduce these gaps. This study evaluated a QI intervention (QPulse) that focussed on CVD assessment and management.

Methods

This mixed-methods study explored the implementation of guidelines and adoption of QI processes in 34 general practices. CVD screening and management were measured pre- and post-intervention. Qualitative analyses examined participants' Plan-Do-Study-Act (PDSA) goals and in-depth interviews with practice stakeholders focussed on barriers and enablers to implementation and were analysed thematically using Normalisation Process Theory (NPT).

Results

Pre- and post-intervention data were available from 15 practices ( $n=19562$ and $n=20249$, respectively) and in-depth interviews from seven practices. At baseline, $45.0 \%$ of patients had their BMI measured and $15.6 \%$ had their waist circumference recorded in the past 2 years and blood pressure, lipids and smoking status were measured in $72.5 \%, 61.5 \%$ and $65.3 \%$ of patients, respectively. Most highrisk patients (57.5\%) were not prescribed risk-reducing medications. After the intervention there were no changes in the documentation and prevalence of risk factors, attainment of BP and lipid targets or prescription of CVD risk-reducing medications. However, there was variation in performance across practices with some showing isolated improvements, such as recording waist circumference (0.7$32.2 \%$ pre-intervention to $18.5 \%-69.8 \%$ post-intervention), BMI and smoking assessment. Challenges to implementation included: lack of time, need for technical support, a perceived lack of value for quality improvement work, difficulty disseminating knowledge across the practice team, tensions between the team and clinical staff and a part-time workforce.

Conclusion

The implementation barriers associated with this QI program was considerable in Australian GP practices. Findings highlighted they were not able to effectively operationalise the intervention due to numerous factors, ranging from lack of internal capacity and leadership to competing demands and insufficient external support.

Trial registration

Australian New Zealand Clinical Trials Reference Number (ACTRN12615000108516), registered 06/02/2015. Trial protocol can be accessed at: https://www.anzctr.org.au/Trial/Registration/TrialReview.aspx?ACTRN=12615000108516

\section{Background}

Cardiovascular diseases (CVD) are the leading cause of death worldwide, despite major declines in morbidity and mortality over the last 40 years (1). In 2015, CVD was responsible for $29 \%$ of deaths, and over 1.1 million hospital admissions in Australia $(2,3)$. CVD burden can be reduced through risk-factor modification (4). Around two-thirds of Australians have three or more modifiable risk factors such as tobacco smoking, high blood pressure (BP) or cholesterol, physical inactivity, poor nutrition, or overweight/obesity (1, 5). Most international guidelines recognise that these risk factors collectively contribute to an individual's overall or 'absolute' risk, and that management decisions should be based on multiple risk factors (5-7). In 2012, the National Vascular Disease Prevention Alliance launched Australia's first absolute risk-based management guideline bringing together several guidelines into a single cohesive approach (5).

General Practitioners (GPs) play a major role in mitigating CVD morbidity and mortality, and see over 85\% of the population (approx. 20 million consultations) in Australia annually (8). However, studies have shown sub-optimal assessment and management of CVD risk. A 2006 study of The Bettering the Evaluation and Care of Health (BEACH) data found that patients at high risk of CVD were substantially under-treated (9). In 2010, the AusHEART study found only $34 \%$ of patients at high CVD risk were prescribed both a BPlowering medication and a statin (10) and with 2011/12 National Health Survey data Banks et al showed that almost half (47.1\%) of high-risk patients were not taking any guideline-recommended medications (4). In 2012, baseline data from the TORPEDO 
randomised controlled trial found that only $48 \%$ of patients were appropriately screened for CVD risk (11), and similar findings were documented in a more recent review of Australian data from 2015-18 that showed 47.9\% of patients had CVD risk screening and only $41 \%$ of high-risk patients were prescribed risk-reducing medications (12).

Quality Improvement (QI) initiatives in primary care have the potential to improve uptake of evidence-based practices (13). QI is a multi-dimensional concept, which can be defined as having a systematic approach to making changes that will lead to better patient outcomes (health), enhanced system performance (care) and improved professional development (learning) (14). There are several ways to intentionally implement QI initiatives, with one approach being the establishment of a Quality-Improvement Collaborative (QIC). QICs bring together groups of practitioners from different 'organisations' to learn about a specific aspect of health service quality, and to share experiences about making changes in their local settings. There has been mixed evidence of success implementing QICs in health care $(15,16)$. However, a systematic review of 64 QIC programs in 2018 reported significant improvements in $83 \%$ of targeted clinical processes and patient outcomes (17).

The current intervention applied a QIC approach to improving CVD management in the 'real world' of Australian general practice in one Primary Health Network (PHN) in Sydney, Australia. Study aims were to: (1) assess whether implementation of a brief QIC approach was associated with improvements in the monitoring, prescribing practices and attainment of BP and lipid targets for CVD risk reduction; and (2) understand barriers and factors driving implementation and adoption of the QIC.

\section{Methods}

A mixed-methods sequential study design utilised data from the QPulse study (Central and Eastern Sydney General Practice Quality Improvement Network: building a sustainable model of QI to achieve reduced cardiovascular disease in the primary care setting) (12, 18).

\section{General practice recruitment}

Practices were recruited from the geographical catchment of Central and Eastern Sydney Primary Health Network (Australia) between May 2015 and November 2016. Practice recruitment is outlined in Fig. 1. PHNs are federally funded meso-tier organisations tasked with supporting the primary health care system, commissioning of services, and working collaboratively to integrate health services within their region. A number of methods were employed to recruit practices, including targeting those that had participated in other QI programs, informal invitation at professional development or local network meetings, and formal invitation via email, newsletters and/or weekly fax communiques to GPs and practice managers (PM).

Practices were eligible if they used one of two electronic medical record software programs (i.e. Medical Director ${ }^{\mathrm{TM}}$ or Best Practice ${ }^{\mathrm{T} \omega}$ ) for recording risk-factor information, pathology results and prescriptions. There was a total of 127 general practices from a possible 670 in the CESPHN catchment approached to participate. Reasons given to the PHN team from 41 of invited non-participating practices included: not enough time, staffing limitations, competing priorities, CVD not being a practice priority area, and not wanting to participate in research.

There were three waves of recruitment between April 2015 - July 2017; twenty-five practices were included in Wave 1, nine in Wave 2 and nine in Wave 3. Wave 3 was subsequently cancelled due to lack of PHN staff resources, and consequently only practices from Waves 1 and $2(n=34)$ participated, with three withdrawing. Complete pre- and post-intervention data were available from fifteen GP practices. In sixteen practices follow-up data were not successfully extracted using the automated data extraction software due to a technical problem in the extraction of prescribed medications from some of the practice records (see Fig. 1). The practices included in the study covered the geographic area of the PHN, represented practices with different billing structures (i.e. bulk- and private-billing) and included both independent- and corporate-owned practices.

The baseline patient characteristics of the 19 services with only pre-intervention data were similar to the 15 with complete (i.e. preand post-intervention) data (Supplementary Table 1). There were no differences in baseline patient demographic, CVD assessment and risk factor profiles between the two samples.

\section{Quality improvement intervention}


The QIC was designed to be overseen and coordinated by CESPHN to complement previous quality improvement projects and utilise existing relationships between PHN staff and the general practices (1). The QPulse project ran over 22 months (May 2015 - February 2017). Participating practices provided the PHN with baseline, de-identified patient data of 'regularly attending' patients (defined below). The PHN generated feedback reports at the commencement of the intervention (using baseline data) for each practice targeting the CVD measurable goals.

At least one stakeholder from each practice, such as a practice nurse, senior GP or PM attended three 2-hour workshops over three weeks. Topics covered: CVD risk assessment tools, absolute risk calculators, current guidelines for preventive CVD management including pharmacological management of high-risk patients, QI theory and specifics on how to achieve change using the Plan Do Study Act (PDSA) methodology (19). By planning a change, trying it, observing the results, and acting on what was learned, participants were guided through a rapid feedback exercise to generate change ideas for their practice. Depending on their specific aim, practice teams were asked to choose ideas to test on a small scale and refine the change as necessary before implementing successful ideas more broadly. Each practice team was asked to submit a monthly PDSA report to the PHN to assist them in planning and measuring change.

During the workshops, participants were also trained in the use of two quality-improvement software tools supplied to each practice: (1) a desktop 'point of care' tool, 'HealthTracker'; a software application that works in conjunction with the electronic medical record to identify high-risk patients and provides GPs with 'real time' personalised guideline recommendations pertaining to cardiovascular risk reduction; and (2) PenCAT Clinical Audit Tool ${ }^{T M}$ a data extraction tool installed onto the computer server at each Practice. PenCAT enabled practices to download specific audit reports containing de-identified patient data on demographics, CVD disease statistics and prescribing information. A data extraction could be performed as frequently as practice staff desired to assist with the PDSA process and was the basis of the monthly data report sent to the PHN.

Members of each general practice were also invited to attend monthly webinars after the initial three workshops to collaboratively share their progress and ideas for change with other participating practice teams. Each practice was offered external support from the PHN to assist with provision and interpretation of the personalised practice report, formulating the PDSAs, and submitting monthly data extractions.

\section{Target population}

Eligible patients were those recommended by guidelines for CVD risk assessment (5), and who were 'regular attenders' at the practice. This included Aboriginal and Torres Strait Islander people $\geq 35$ years and all others $\geq 45$ years; and those at clinically high risk of CVD regardless of age (defined below). 'Regular attenders' were defined as patients who attended the practice at least three times in the previous 24 months, and at least once in the previous 6-month period.

Five-year CVD risk was calculated using the 1991 Anderson Framingham Risk equation using sex, age, systolic blood pressure, smoking status, total cholesterol, high density lipoprotein (HDL) cholesterol and diabetes status (20). Diabetes and left ventricular hypertrophy were assumed to be absent, unless explicitly recorded as diagnoses in the patient record. As per current guidelines, high CVD risk was defined as any of the following: (i) calculated 5-year CVD risk exceeding 15\% based on the FRE, (ii) presence of clinically high-risk conditions (including diabetes and age $>60$ years, diabetes and albuminuria, eGFR $<45 \mathrm{ml} / \mathrm{min} / 1.73 \mathrm{~m}^{2}$, systolic BP > $180 \mathrm{mmHg}$, diastolic $\mathrm{BP}>110 \mathrm{mmHg}$ or total cholesterol $>7.5 \mathrm{mmol} / \mathrm{L}$ ) (iii) presence of a CVD diagnosis (i.e. coronary heart disease, cerebrovascular disease, peripheral vascular disease). (5)

\section{Outcome measures}

Outcome measures were: (1) CVD risk factor assessment including BP assessment in the past year, and lipid, BMI, waist circumference and smoking status assessment in the past 2 years; (2) guideline-recommended treatment for people defined as prescription of a BP-lowering medication and a statin for people at high CVD risk and prescription of a BP-lowering medication, a statin and either an antiplatelet or an anticoagulant agent for people with a diagnosis of existing CVD (see Appendix A for specific medications); and (3) meeting CVD targets, which were defined as a BP less than $140 / 90 \mathrm{mmHg}$ for high-risk patients, and less than $130 / 80 \mathrm{mmHg}$ for those with established CVD or diabetes and a total cholesterol $<4.0 \mathrm{mmol} / \mathrm{L}$, high-density lipoprotein (HDL) level of $>1.0 \mathrm{mmol} / \mathrm{L}$, low-density lipoprotein (LDL) level of $<2.0 \mathrm{mmol} / \mathrm{L}$.

\section{Statistical analyses}


Bivariate analyses examined the frequencies and proportions of patients by their sociodemographic, CVD risk, BP/lipid levels and prescription of risk-reducing medications. Logistic regression models examined differences between baseline and post-intervention in the key outcome variables with adjustment for gender, age and Indigenous status. Changes between baseline and post-intervention were assessed by odds ratios $(95 \% \mathrm{Cls})$, using the baseline sample as the reference category. As the data were hierarchical (i.e. patients clustered within GP practices), all analyses were adjusted for clustering at the practice level and applying finite population correction when estimating variance. Analyses were conducted with SPSS statistics software Version 26.0.0. Statistical significance was considered as $p<0.05$ (two-tailed).

\section{Qualitative data analysis}

The PDSAs submitted by each practice were analysed by thematic analysis. The goals addressed in the PDSAs were coded by two coders who worked independently. Coders then sorted the themes of the PDSAs into larger categories and then discussed their results, examined any discrepancies and reached consensus for a final coding classification. Following this, 19 semi-structured interviews were conducted after completion of the intervention with a purposive sample of people involved in QPulse implementation, including PMs, nurses and GPs, as well as project officers, IT support personnel and managers at the PHN (Table 1). The interview guide is provided in Appendix B. 
Table 1

Pre- and post-intervention data of sociodemographic, cardiovascular risk assessment and management in QPULSE

\begin{tabular}{|c|c|c|c|c|c|c|c|c|}
\hline & \multicolumn{4}{|l|}{ Pre-intervention } & \multicolumn{4}{|l|}{ Post-intervention } \\
\hline & \multicolumn{3}{|c|}{ Cardiovascular risk assessment } & \multirow{2}{*}{$\begin{array}{l}\text { Total } \\
\text { sample } \\
\text { in } 15 \\
\text { practices } \\
(n=19 \\
562)\end{array}$} & \multicolumn{3}{|c|}{ Cardiovascular risk assessment } & \multirow{2}{*}{$\begin{array}{l}\text { Total } \\
\text { sample } \\
\text { in } 15 \\
\text { practices } \\
(n=20 \\
249)\end{array}$} \\
\hline & $\begin{array}{l}\text { Low/moderate } \\
(n=8171)\end{array}$ & $\begin{array}{l}\text { High } \\
(\mathrm{n}= \\
1139)\end{array}$ & $\begin{array}{l}\text { Established } \\
\text { CVD } \\
(n=2372)\end{array}$ & & $\begin{array}{l}\text { Low/moderate } \\
(n=9155)\end{array}$ & $\begin{array}{l}\text { High } \\
(n= \\
1195)\end{array}$ & $\begin{array}{l}\text { Established } \\
\text { CVD } \\
(n=2537)\end{array}$ & \\
\hline Age (mean, SD) & $61.1(11.2)$ & $\begin{array}{l}72.4 \\
(9.2)\end{array}$ & $73.6(11.3)$ & $\begin{array}{l}63.9 \\
(12.5)\end{array}$ & $60.8(11.1)$ & $\begin{array}{l}71.9 \\
(9.2)\end{array}$ & $73.5(11.4)$ & $\begin{array}{l}63.8 \\
(12.4)\end{array}$ \\
\hline \multicolumn{9}{|l|}{ Gender } \\
\hline Male & $38.2(3124)$ & $\begin{array}{l}78.7 \\
(896)\end{array}$ & $62.9(1491)$ & $\begin{array}{l}44.6 \\
(8717)\end{array}$ & $38.5(3525)$ & $\begin{array}{l}78.4 \\
(937)\end{array}$ & $61.8(1569)$ & $\begin{array}{l}55.5 \\
(11236)\end{array}$ \\
\hline Female & $61.8(5047)$ & $\begin{array}{l}21.3 \\
(243)\end{array}$ & 37.1 (879) & $\begin{array}{l}55.3 \\
(10816)\end{array}$ & $61.5(5630)$ & $\begin{array}{l}21.6 \\
(258)\end{array}$ & $38.1(967)$ & $\begin{array}{l}44.5 \\
(9013)\end{array}$ \\
\hline Missing & & & & $0.1(29)$ & & & & $0.1(13)$ \\
\hline \multicolumn{9}{|l|}{ Ethnicity } \\
\hline $\begin{array}{l}\text { Aboriginal or } \\
\text { Torres Strait } \\
\text { Islander }\end{array}$ & $0.4(30)$ & $0.5(6)$ & $0.3(6)$ & $0.4(77)$ & $0.4(35)$ & $0.7(8)$ & $0.5(12)$ & $0.5(109)$ \\
\hline Other & $99.6(8141)$ & $\begin{array}{l}99.5 \\
(1133)\end{array}$ & $99.7(2366)$ & $\begin{array}{l}99.6 \\
(19485)\end{array}$ & $99.6(9120)$ & $\begin{array}{l}99.3 \\
(1187)\end{array}$ & $99.5(2525)$ & $\begin{array}{l}99.5 \\
(20153)\end{array}$ \\
\hline \multicolumn{9}{|l|}{$\begin{array}{l}\text { Risk factor } \\
\text { assessment }\end{array}$} \\
\hline Blood pressure ${ }^{a}$ & $81.9(6690)$ & $\begin{array}{l}86.1 \\
(981)\end{array}$ & $81.0(1922)$ & $\begin{array}{l}72.5 \\
(14175)\end{array}$ & $85.9(7862)$ & $\begin{array}{l}93.0 \\
(1111)\end{array}$ & $83.5(2119)$ & $\begin{array}{l}76.4 \\
(15487)\end{array}$ \\
\hline Blood lipids $^{b}$ & $82.5(6739)$ & $\begin{array}{l}82.0 \\
(934)\end{array}$ & $70.7(1678)$ & $\begin{array}{l}61.5 \\
(12026)\end{array}$ & $85.0(7781)$ & $\begin{array}{l}86.5 \\
(1034)\end{array}$ & $75.5(1916)$ & $\begin{array}{l}65.9 \\
(13361)\end{array}$ \\
\hline $\mathrm{BMI}^{\mathrm{b}}$ & $54.6(4460)$ & $\begin{array}{l}57.4 \\
(654)\end{array}$ & 49.4 (1171) & $\begin{array}{l}45.0 \\
(8814)\end{array}$ & $59.0(5394)$ & $\begin{array}{l}65.4 \\
(782)\end{array}$ & 45.1 (1347) & $\begin{array}{l}49.4 \\
(10000)\end{array}$ \\
\hline $\begin{array}{l}\text { Waist }^{\mathrm{b}} \\
\text { circumference }^{\mathrm{b}}\end{array}$ & 21.0 (1719) & $\begin{array}{l}25.3 \\
(288)\end{array}$ & 15.9 (377) & $\begin{array}{l}15.6 \\
(3039)\end{array}$ & $25.3(2314)$ & $\begin{array}{l}32.4 \\
(387)\end{array}$ & 19.9 (506) & $\begin{array}{l}34.7 \\
(3952)\end{array}$ \\
\hline Smoking status & 63.2 (5164) & $\begin{array}{l}70.1 \\
(798)\end{array}$ & 71.1 (1686) & $\begin{array}{l}65.3 \\
(12774)\end{array}$ & $63.9(5785)$ & $\begin{array}{l}65.3 \\
(780)\end{array}$ & 66.8 (1695) & $\begin{array}{l}65.7 \\
(13304)\end{array}$ \\
\hline \multicolumn{9}{|l|}{ BMI } \\
\hline Underweight & $3.2(260)$ & $\begin{array}{l}1.1 \\
(13)\end{array}$ & $2.1(51)$ & $2.5(484)$ & $3.4(310)$ & $\begin{array}{l}0.8 \\
(10)\end{array}$ & $2.6(65)$ & $2.7(541)$ \\
\hline Healthy weight & 21.6 (1768) & $\begin{array}{l}13.6 \\
(155)\end{array}$ & $14.2(336)$ & $\begin{array}{l}16.8 \\
(3282)\end{array}$ & $23.1(2114)$ & $\begin{array}{l}15.8 \\
(189)\end{array}$ & $15.2(386)$ & $\begin{array}{l}18.1 \\
(3675)\end{array}$ \\
\hline
\end{tabular}

A Assessed in the past 12 months.

B Assessed in the past 2 years.

C 'At risk' waist circumference $>94 \mathrm{~cm}$ for males and $>80 \mathrm{~cm}$ for females.

D Guideline-recommended treatment was defined as (1) For high-risk patients the prescription of a BP-lowering medication and a statin; [5] for patients with established CVD, prescription of a BP-lowering medication, a statin and either an antiplatelet or an anticoagulant agent (see Appendix A for specific medications). 


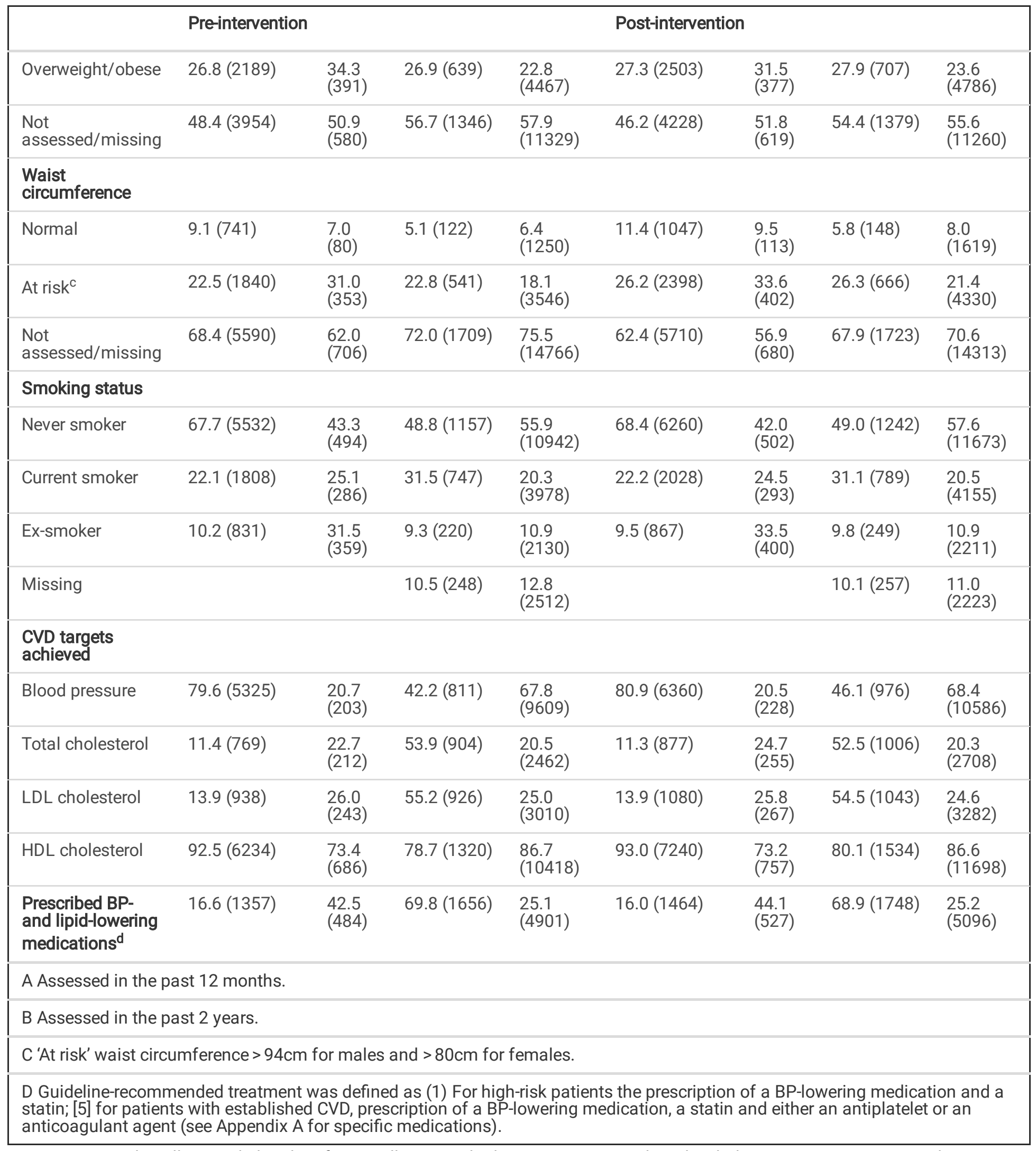

Interviews were digitally recorded and professionally transcribed. Transcripts were shared with the interviewees to ensure they were accurate, and that they were agreeable to the contents being used for the study. Four researchers independently read and analysed the interview transcripts; this included the principal investigator and three researchers who had not taken part in the QPulse study. Interviews were manually coded by each researcher guided by the domains in Normalisation Process Theory (NPT). The findings were iteratively reviewed and refined at two group meetings with the principal investigator and three researchers. (21) 
NPT is a theory of implementation designed to aid interpretation of how interventions are embedded, enacted and operationalised within healthcare settings. This approach assumes four main generative mechanisms (coherence or sense making; cognitive participation, collective actions; and reflexive monitoring) which are needed to achieve change in practice. The aim of the analysis was to examine the 'real world' participant perceptions of, and responses to the multicomponent QI intervention, and at which point in implementation the intervention was perceived to be sustainable or have failed.

This research was performed in accordance with the Declaration of Helsinki and was approved by the University of Notre Dame Australia Human Research Ethics Committee (HREC) (reference 014105S). Signed agreements with participating practices were also obtained. A consent waiver for patient-level consent was granted by the committee.

\section{Results}

\section{Practice characteristics and engagement}

Of the 15 practices with complete pre-and post-data, all attended at least one workshop, 11 attending two and six attended all three workshops. Most practices sent only one attendee to each workshop with three practices sending two attendees and one practice sending three attendees to all three workshops. The PHN recorded contact with all participating practices at least once a month, some requested higher levels of interaction (range 1 to 8 contacts per month) which was provided via phone or face-to-face, to help with IT and QI processes. The project officer recorded a median of four practice visits and 15 phone calls per practice over the duration of the project. Although 12 practices registered to attend the first two webinars, only two attended, and these were consequently discontinued after two months. All participating practices submitted a baseline PDSA.

\section{Baseline patient characteristics}

The mean age of patients at baseline was 63.9 years, and the majority $(55.3 \%, n=10816)$ were female. $5.8 \%(n=1139)$ had high absolute cardiovascular disease or high clinical risk, and 12.1\% $(n=2372)$ already had a diagnosis of CVD (Table 1). A greater proportion of patients at high risk of CVD or established CVD had up-to-date BP, lipid, BMI and waist circumference measures than those with low/moderate risk. High-risk patients were more likely to be overweight/obese or current smokers than their low/moderate risk counterparts. They were also more likely to reach total cholesterol and LDL targets, however a lower proportion met BP and HDL targets. A minority (41.2\%) of high-risk patients were prescribed recommended medication, whereas a majority (69.5\%) of those with established CVD were prescribed the recommended risk-reducing medications.

\section{Post- QI intervention}

The sociodemographic profile of patients in the post-intervention sample was similar to the baseline characteristics, as were the CVD risk factor profiles, screening and management (Table 1). Post-intervention cardiovascular risk assessment, risk profiles and CVD targets were compared with baseline in Table 2. There were no significant improvements in any of the outcomes post-intervention, however there was wide variation in practice performance. Supplementary Table 2 provides summaries of the results of four highperforming practices where marked changes in selected outcomes were observed, and showed improvements in the recording of BMI in Practices 1 and 2 after the intervention. Practices 1-3 also showed large improvements in the measurement of waist circumference, whereas Practice 4 had comparatively high baseline levels of all risk factor assessment and showed no overall change in these measures post-intervention. 
Table 2

Changes in CVD risk factor assessment, CVD targets and prescribing behaviours post-intervention compared to baseline

OR ( $95 \% \mathrm{Cl})$ post-intervention compared to baseline

\begin{tabular}{llll} 
Total & \multicolumn{2}{l}{ Cardiovascular risk assessment level post-intervention } \\
\cline { 2 - 3 } $\begin{array}{l}\text { post-intervention } \\
\text { sample }\end{array}$ & Low/moderate & High & Established CVD \\
in 15 practices & $(n=17365)$ & $(n=2334)$ & $(n=4975)$ \\
$(n=40256)$ & & &
\end{tabular}

\section{Risk factor assessment}

\begin{tabular}{|c|c|c|c|c|}
\hline Blood pressure & $1.23(0.90-1.70)$ & $\begin{array}{l}1.36(0.82- \\
2.25)\end{array}$ & $1.12(0.85-1.48)$ & $1.22(0.80-1.86)$ \\
\hline Blood lipids ${ }^{b}$ & $1.22(0.94-1.58)$ & $\begin{array}{l}1.21(0.75- \\
1.95)\end{array}$ & $1.40(0.56-3.49)$ & $1.29(0.93-1.79)$ \\
\hline $\mathrm{BMI}^{\mathrm{b}}$ & $1.19(0.99-1.42)$ & $\begin{array}{l}1.19(0.95- \\
1.50)\end{array}$ & $1.40(0.90-2.19)$ & $1.17(0.93-1.46)$ \\
\hline Waist $^{b}$ circumference $^{b}$ & $1.32(0.74-2.35)$ & $\begin{array}{l}1.27(0.61- \\
2.65)\end{array}$ & $1.41(0.81-2.45)$ & $1.34(0.88-2.03)$ \\
\hline Smoking status ${ }^{b}$ & $1.17(0.91-1.48)$ & $\begin{array}{l}1.13(0.91- \\
1.38)\end{array}$ & $1.09(0.75-1.38)$ & $1.11(0.88-1.38)$ \\
\hline \multicolumn{5}{|l|}{ CVD risk factors } \\
\hline Overweight/obesity & $0.97(0.94-1.00)$ & $\begin{array}{l}0.96(0.91- \\
1.01)\end{array}$ & $0.80(0.63-1.02)$ & $1.00(-.90-1.11)$ \\
\hline Waist circumference ${ }^{c}$ & $1.02(0.68-1.53)$ & $\begin{array}{l}0.99(0.62- \\
1.60)\end{array}$ & $0.86(0.61-1.22)$ & $1.07(0.73-1.55)$ \\
\hline Current smoker & $0.99(0.94-1.05)$ & $\begin{array}{l}0.91(0.81- \\
1.02)\end{array}$ & $1.05(0.98-1.13)$ & $1.05(0.92-1.20)$ \\
\hline
\end{tabular}

\section{CVD targets achieved}

\begin{tabular}{|c|c|c|c|c|}
\hline Blood pressure & $1.02(0.90-1.16)$ & $\begin{array}{l}1.08(0.97- \\
1.21)\end{array}$ & $0.99(0.70-1.40)$ & $1.16(1.02-1.33)$ \\
\hline Total cholesterol & $0.99(0.92-1.06)$ & $\begin{array}{l}0.99(0.91- \\
1.08)\end{array}$ & $1.15(0.90-1.46)$ & $0.95(0.84-1.07)$ \\
\hline LDL cholesterol & $0.98(0.92-1.04)$ & $\begin{array}{l}1.00(0.94- \\
1.06)\end{array}$ & $1.00(0.83-1.20)$ & $0.98(0.87-1.11)$ \\
\hline HDL cholesterol & $1.08(0.99-1.18)$ & $1.09(1.00-1.20)$ & $0.99(0.84-1.13)$ & $1.08(0.95-1.23)$ \\
\hline \multicolumn{5}{|c|}{ Prescribed CVD risk-reducing medications } \\
\hline $\begin{array}{l}\text { Prescribed risk-reducing } \\
\text { medication(s) }\end{array}$ & - & - & $1.07(0.97-1.19)$ & $0.96(0.91-1.02)$ \\
\hline
\end{tabular}

A Assessed in the past 12 months.

B Assessed in the past 2 years.

C 'At risk' waist circumference $>94 \mathrm{~cm}$ for males and $>80 \mathrm{~cm}$ for females.

D Guideline-recommended treatment was defined as (1) For high-risk patients the prescription of a BP-lowering medication and a statin; [5] for patients with established CVD, prescription of a BP-lowering medication, a statin and either an antiplatelet or an anticoagulant agent (see Appendix A for specific medications).

\section{PDSA themes}


Every practice submitted at least one PDSA with only one practice submitting monthly, as requested. Overall, the PDSAs had an emphasis on foundational goals such as improving data measurements (e.g. recording of waist circumference or smoking status) rather than focusing on changes in preventive care and guideline-based prescribing (neither lifestyle nor medication). In some cases, PDSA goals aligned with improvements in practice data as seen in one practice (Fig. 2) that focused on waist circumference measurement, however these were observed in a minority of the more engaged practices.

\section{Interview analysis}

The characteristics of interview participants at the PHN and practice levels are shown in Table 3. There were a range of stakeholders interviewed within various roles and levels of the organisations, with a good distribution of participants in practices are varying size and with prior QI experience. The interviews yielded rich insights into understanding the quantitative findings. Multiple barriers to implementation across all four NPT domains were described and summarised below.

Table 3

Interviewee characteristics from the Primary Health Networks (PHNs) and from general practices

\begin{tabular}{|lc|}
\hline Interview participants from PHNs $(\mathbf{n}=\mathbf{7})$ & 4 \\
\hline Female & 2 \\
\hline Project Officer & 2 \\
\hline Team Manager & 1 \\
\hline Executive Officer & 2 \\
\hline IT Support Officer & $\mathbf{n}=\mathbf{1 2})$ \\
\hline Interview participants from general practices & 9 \\
\hline Female & 1 \\
\hline Practice nurse & 1 \\
\hline Practice manager & 10 \\
\hline General Practitioner & \\
\hline Practice size & 1 \\
\hline (number of regular patients) & 1 \\
\hline$<2000$ & 3 \\
\hline $2001-4000$ & 3 \\
\hline $4001-6000$ & 2 \\
\hline $6001-8000$ & 1 \\
\hline $8001-10000$ & 1 \\
\hline $10001-20000$ & 6 \\
\hline$>20001$ & 1 \\
\hline Previous QI experience & \\
\hline No previous QI experience & \\
\hline
\end{tabular}

\section{Coherence (the meaning ascribed individually and collectively to a new set of practices)}

Collectively, GPs and PHN staff saw the potential value of the intervention as very high, for example: "There are a whole lot of people at risk, and they could have better outcomes...." (GP6). Access to the education modules and the two QI tools were seen as useful in 
identifying and providing preventive care guidelines to high-risk patients. Only one participant mentioned that internal leadership ('Change Champion') or practice support ('QI Culture') was needed to facilitate engagement with the intervention.

\section{Cognitive participation (commitment to engage with the new)}

Interviewees from both PHNs and practices noted that the timing of this project (coinciding with major PHN governance and strategy change) prohibited prioritisation of support for the intervention by PHN Staff.

Most interviewees reported they had not fully appreciated what participation in a QI project would require of them prior to the intervention, "... when I took it on I didn't realise there was more to it, so I didn't really understand...". (GP1). For PHN participants this was illustrated at the highest executive level where management under-estimated the readiness/ability of practices to be enrolled in this project, along with the need for the PHN to supply adequate resourcing to support both their staff and the participant practices. "With 40 practices enrolled, the workload sometimes got overwhelming despite team members help(ing) me with 6 of the practices". (PHN3) This lack of strategic planning and resourcing was amplified by the change in focus of PHN staff and a merger of three earlier meso-tier organisations into a single PHN. This affected directly on the PHN's ability and commitment to supply practice level support during the intervention period.

GP engagement with the PDSA process was also extremely low. While the PDSAs were understood by most GPs as an essential part of the QI process, they were seen as time-consuming, and "... formulaic.... uninteresting..." (GP1) and "...to be honest, no, I haven't done one since we started." (GP9) with only a few seeing value in this aspect of the project. "It is a problem to stay on track and keep getting things done... I had a million good intentions, and then it gets ...too hard". (P4)

\section{Collective action (how the work does/not get done)}

Participants reported a lack of both 'QI culture' and change champions to support engagement with QPulse. Although there was universal agreement that 'key individuals were needed to drive the intervention forward', this did not mean that these individuals were found, nor engaged to help with the project. "...it comes down to the culture within the practice, who is the real leader...the driver in the practice. It could be a nurse or the doctor... but crucially you really need to have somebody who is going to take the reins, or it doesn't happen...." (PHN5). It was clear many participants were unable to actively champion or drive the project forward due to not taking on a leadership role within their practice setting. Differing practice systems often meant each GP within the practice worked as an individual rather than part of a cohesive system of care. "It's quite individual. That's the way the practice is set up.... your quality control is up to you.... As long as it doesn't add any extra work... because no-one obviously is interested if it's extra work." (GP2)

Communication systems between GPs, nurses and PMs were cited as a barrier to engagement. Often there was no regular practice 'team' meetings or systems in place to report back about QI measures and limited ability to organise tailored educational activities. Jobs were delegated to non-medical staff (e.g. the PM or nurse) who may/not have the skills or motivation to drive the project forward due to a lack of personal engagement with the goals of the project. At one practice, a manager commented that she only became involved because: (the GP) "... didn't want to do it, so she handed it to me... I actually didn't know much about it." (GP12). In addition, GP attendance at the educational sessions was sub-optimal and inconsistent, with many GPs delegating this to the PM or nurse. The scheduled monthly networking/seminar meetings were cancelled due to lack of attendance, despite most participants opting to join at the beginning of the project.

There was commentary that the QI project and data reports did not address key issues around improving engagement with patients: "It's one thing to get the GPs to change what they do, it's an entirely different thing to get patients to take in on board." (GP 6) Several GPs also discussed the problem with many competing projects and lack of time: "I think the difficulty is there's a plan for one quality of project and then another idea comes up and then the same people are looking at implementing it, or we give the nurses something else to do, and it sort of falls off the radar." (GP6)

Pparticipants reported barriers to setting up a sustainable QI processes. This was highlighted by commentary about the difficulty of scheduling data extractions and generation of reports, low attendance at QI educational activities, no engagement with PDSA process, no evidence of sustained use of the IT tools. Several attributed this to both lack of dedicated time to do QI work and lack of any tangible incentives (financial or professional development). Many reported that GPs were not keen to engage with an activity that was 
not aligned with financial incentives and cited such incentives as a mechanism to achieve long-term engagement rather than as the first reason for engagement. However, engagement with individual "contracted" GPs was reported as requiring a financial incentive to engage them with doing any of the extra work involved in QI activities. "... from a practice perspective, it's not going to be a priority. So really, the PHN needs to take on a lot of that responsibility on behalf of the practice if we're going to get it up and running". (PHN1)

Minor IT issues were also identified as barriers, although it was well understood by participants that IT support was readily available if asked: "I used it for a couple of weeks and found it was really useful... (when a minor IT issue arose to render the tool inactive) ... it just kind of died off, my use of it." (GP7) Finally, it was noted that the intervention did not adequately accommodate the roles of individual GPs (and patients) in achieving key outcomes (such as BP, lipids levels) and medication prescriptions. "It was all a bit clunky...I never saw the Manager's data reports... and although I tried with Q Pulse, ... if a doctor is not interested, they won't do it ...that's often the way around here and I just couldn't engage them with recording the CVD measures." (GP2)

PHN staff reported a lack of resourcing to provide individualised GP practice reports, education or face-to-face support despite observing that most practices needed significantly more support than anticipated to complete the basic requirements of the program (such as monthly data extractions, PDSAs, tidying up eMR systems). "Until you start giving monthly reports and with targeted topics and actually educating the GPs about what to do with that data, you're just extracting data". (PHN1)

\section{Reflexive monitoring (the processes through which practitioners decide whether new approaches are beneficial and lends, ultimately, to the normalisation of new practices)}

Normalisation of systematised QI practices was not apparent, even among the most experienced and engaged practices. This was despite many participants reporting enthusiasm for ongoing participation in QI work.. "So, we've talked about it, but we've never implemented it systematically... most doctors are not taught these things, that's the whole problem, so we've got to re-educate the doctors, our universities haven't got it in the curriculum." (GP7)

Even in solo practices, GPs experienced challenges with translating the project QI goals into long-term changes systematic CVD preventive care. The project learnings were discussed as a one-off piece of work rather than something to embed into everyday routine practice. "It's the follow through which can be difficult because you will forget. I don't know that this is common to everyone, but ...if somebody is not ...pestering you and reminding you... - I've actually forgotten how to do it - it falls off the wagon." (GP3)

The challenges associated with achieving collective action for both general practices and the PHN meant participants found it difficult to move beyond 'new' and toward 'normal'. Interviewees reported a 'project-based' approach to QI interventions with 'topical' engagement and difficulty setting up systematised adoption of change due to the considerable number of competing projects.

Some GPs reported adopting new ways of approaching CVD preventive care, such as utilising CVD absolute risk assessment tools or using audit data extraction tools to identify gaps in data measures such as smoking status, BMI or waist circumference, "every single patient I see I just flick onto the summary screen of HealthTracker and just see whether the percentage is something I need to worry about, and if it's not, then I don't pursue it." (GP4) However, few mentioned systematic use of the tools for the whole team. Even the most engaged general practice participants noted a difficulty in systematising QI in the day-to-day running of business. "I think (the workplace of) general practice is a barrier, it's an unpredictable, busy, chaotic job and so things happen that get in the way. And I think the other barrier is that protected time, to set up systems is an enabler and not being in place is a barrier". (GP6)

\section{Discussion}

Engagement with the QPulse QI intervention was limited in most general practices. Pre-intervention data showed sub-optimal assessment of CVD risk factors, along with low prescribing rates among high-risk patients and after the intervention there were no significant changes in the assessment of CVD risk factors, the achievement of BP and blood lipid targets or the prescription of riskreducing medications. Overall, GPs considered the intervention as relevant and beneficial to patient care. Stakeholders identified that activity was mainly led by non-medical staff and focussed on process measures rather than clinical outcomes. There were substantial challenges for GPs for embedding the intervention into long-term changes in clinical practice.

It is difficult to ascertain whether these were due to limitations in the design of the intervention or in its implementation, however given the limited success of many interventions in primary care $(11,22,23)$ it is likely that implementation played a larger role. Primary

Page $12 / 18$ 
health care is a complex environment that benefits from structured systems of care to aid the adoption of best practice. The privatised model of Australian general practice, which rewards clinicians for volume-based care is a financial and philosophical barrier to widespread adoption of QI programs which focus on 'value- based' care (18).

At the time of this intervention, there was no systematic funding or incentives for GPs and practices to improve their data collection processes, and such improvements were mainly driven by practice 'champions' and needed to be supported by the systems and IT infrastructure to normalise these changes. These supports were not in place and were identified as key factors limiting implementation in the stakeholder interviews. In many cases, there was no clear role of a leader within practices, and when leadership developed it was often from non-medical staff. Furthermore, this QI intervention was unable to capture doctor-patient decision making around individual choices and the adoption of CVD risk recommendations, including medications, diet and exercise.

The findings also suggest that implementing change needs to build practice capacity and culture. The results showed that even after the QI intervention, a substantial proportion of practices (30-40\%) were not meeting basic documentation and monitoring targets. Improvements have been identified as key to increasing uptake of evidence-based practice in primary care $(13,16)$. This study highlights the need for concerted efforts to set up these foundations at the individual GP, practice and PHN levels to improve the uptake of QI programs.

A qualitative review of QI implementation in Australia identified the need for external support, accurate data and reporting, education and change champions in addition to financial incentives (18). Other government-funded initiatives such as heart health assessments, health assessments for 45-49-year-olds, GP management plans and home medication reviews have similarly only had partial uptake and mixed evidence of success (8) (24). One potential driver of their mixed success is that they are still fee-for-service activities and do not support GPs to adopt quality-focused models of care. The Federal "Health Care Homes" pilot and a proposed voluntary patient enrolment scheme aligned to a patient 'complexity' payment both offer options for value-based care and a departure from the prevailing fee-for-service paradigm. It is still to be seen whether such initiatives will translate to improvements in CVD management and subsequent reductions in disease burden.

While there has been increased interest in supporting QI in primary care, there needs to be a change in thinking from start-up programs to sustained initiatives. A pre-post evaluation of a national QI program, the Australian Primary Care Collaborative program from 2004-2014, found improvements in both CVD risk identification and management, but only had partial uptake nationally (13) and did not receive ongoing Federal funding. Implementation of this program relied on a complex interaction of enablers such as leadership, accurate data, funding incentives, organisational culture, and primary care systems designed to support quality care $(18,22,28)$.

The current study had a number of limitations that need to be acknowledged in the context of the results. Firstly the practices were located in one PHN region and may not be representative of general practices elsewhere. For example, we have previously found higher performance for CVD risk management in Aboriginal Community Controlled Health Services compared with other general practices (10). Secondly, although de-identified electronic medical record data extraction tools allow for a 'whole-of-practice' snapshot of performance, the data need to be entered in extractable fields of the record. This may result in an underestimates of management practices (29). This mainly applies to BP and smoking status, given that electronic prescribing and pathology results are adopted in most Australian general practices. Thirdly, in assessing measurement of absolute CVD risk we looked at the recording of risk factor data but were not able to assess to what extent risk scores were calculated. Given GPs vary greatly in how they use risk scores, the gaps in risk-based management may also vary when using surrogate measures to capture the outcomes of complex GP-patient interactions (30).

\section{Conclusions}

Practitioners see the value in targeting CVD for QI programs however they experience substantial challenges in implementing change if this is not supported more widely. QI programs targeted at the multiple stakeholders and systems working in the primary care setting are required to reach more substantial and sustained process changes, including prescribing practices and risk factor targets, Furthermore, longer-term sustained efforts are needed.

\section{Abbreviations}




\begin{tabular}{ll} 
BMI & Body mass index \\
\hline BP & Blood pressure \\
\hline CVD & Cardiovascular disease \\
\hline GP & General practitioner \\
\hline HDL & High-density lipoprotein \\
\hline LDL & Low-density lipoprotein \\
\hline PDSA & Plan-Do-Study-Act \\
\hline PHN & Primary Health Network \\
\hline QI & Quality improvement \\
\hline QIC & Quality improvement collaboration
\end{tabular}

\section{Declarations}

\section{Ethics approval and consent to participate}

This research was performed in accordance with the Declaration of Helsinki and was approved by the University of Notre Dame Australia Human Research Ethics Committee (HREC) (reference 014105S). Signed agreements with participating practices were also obtained. A consent waiver for patient-level consent was granted by the committee.

\section{Consent for publication}

Not applicable

\section{Availability of data and materials}

The datasets generated and/or analysed during the current study are not publicly available but data may be made available to interested parties from the corresponding author on reasonable request.

\section{Competing interests}

The authors declare that they have no competing interests.

\section{Funding}

The BUPA Health Foundation funded the QPulse project.

\section{Author's Contributions}

$\mathrm{CH}$ made substantial contributions to conception and design of the study, acquisition of funding, conducting interviews of participants, analysis and interpretation of data and drafted the manuscript. DP made a major contribution to the conception and design of the study, as well as acquisition of funding, and analysis and interpretation of the data. MH was a major contributor to conception, acquisition of the funding, design of the study, interpretation of data and drafting of the manuscript. KG was a contributor to the analysis and interpretation of the quantitative data and was involved in drafting and reviewing the manuscript. All authors read and approved the manuscript. 


\section{Acknowledgements}

We would like to thank BUPA Health Foundation for funding the project and for all participating practices and stakeholders for taking part in the study. We would like to acknowledge the Central and Eastern Sydney Primary Health Network (CESPHN) for their endorsement of the project and support for the project via their Quality Improvement project team, oversight of Project Officer, recruitment of practices, provision of practice and IT support, and participation in the interviews. We would also like to acknowledge Dr Denise Campbell, Dr Sarah Wilks and Dr Edwina Brown for data coding and validity checking of the qualitative data analysis for this study.

\section{References}

1. Australian Institute of Health and Welfare. Australia's Health 2018. Canberra: Australian Institute of Health and Welfare; 2018.

2. Australian Institute of Health and Welfare. Primary Health Care in Australia. Canberra: Australian Institute of Health and Welfare; 2016.

3. Australian Institute of Health and Welfare. Cardiovascular Disease Snapshot. Canberra: Australian Institute of Health and Welfare; 2018.

4. Banks E, Crouch SR, Korda RJ, Stavreski B, Page K, Thurber KA, et al. Absolute risk of cardiovascular disease events, and blood pressure-and lipid-lowering therapy in Australia. Med J Aust. 2016;204(8):320.

5. National Vascular Disease Prevention Alliance. Guidelines for the management of absolute cardiovascular disease risk. 2012. http://www.cvdcheck.org.au/pdf/Absolute_CVD_Risk_Full_Guidelines.pdf. Accessed 15 December 2015.

6. Goff Jr D, Lloyd-Jones D, Bennett G, Coady S. D' Agostino RB, Gibbons R, et al. 2013 ACC/AHA guideline on the assessment of cardiovascular risk: a report of the American College of Cardiology/American Heart Association Task Force on Practice Guidelines. Circulation. 2014;129:S49-S73.

7. Heart Foundation of Australia and Cardiac Society of Australia and New Zealand. Reducing risk in heart disease: an expert guide to clinical practice for secondary prevention of coronary heart disease. Melbourne: National Heart Foundation of Australia; 2012.

8. Royal Australian College of General Practitioners. General Practice Health of the Nation (2018): an insight into the state of general practice. Melbourne, Australia: Royal Australian College of General Practitioners; 2018.

9. Webster RJ, Heeley EL, Peiris DP, Bayram C, Cass A, Patel AA. Gaps in cardiovascular disease risk management in Australian general practice. Med J Aust. 2009;191(6):324-9.

10. Heeley EL, Peiris DP, Patel AA, Cass A, Weekes A, Morgan C. Cardiovascular risk perception and evidence-practice gaps in Australian general practice (the AusHEART study). Med J Aust. 2010;192.

11. Peiris D, Usherwood T, Panaretto K, Harris M, Hunt J, Patel B. The Treatment of cardiovascular Risk in Primary care using Electronic Decision suppOrt (TORPEDO) study: intervention development and protocol for a cluster randomised, controlled trial of an electronic decision support and quality improvement intervention in Australian primary healthcare. BMJ Open. 2012;2.

12. Hespe C, Campain A, Webster R, Patel A, Rychetnik L, Harris M, et al. Implementing cardiovascular disease preventive care guidelines in general practice: an opportunity missed. Med J Aust. 2020; 213(7):327-8.

13. Knight A. The collaborative method: a strategy for improving Australian general practice. Aust Fam Physician. 2004;33(4):269.

14. Batalden PB, Davidoff F. What is "quality improvement" and how can it transform healthcare? Qual Saf Health Care. 2007;16(1):2-3.

15. Berwick DM. The Question of Improvement. JAMA. 2012;307(19):2093-4.

16. Knight AW, Caesar C, Ford D, Coughlin A, Frick C. Improving primary care in Australia through the Australian Primary Care Collaboratives Program: a quality improvement report. BMJ Quality \& Safety. 2012:bmjqs-2011-000165.

17. Wells S, Tamir O, Gray J, Naidoo D, Bekhit M, Goldmann D. Are quality improvement collaboratives effective? A systematic review. BMJ Quality \& Safety. 2018;27(3):226-40.

18. Hespe C, Rychetnik L, Peiris D, Harris M. Informing implementation of quality improvement in Australian primary care. BMC Health Services Research. 2018;18(1):287.

19. New South Wales Health Clinical Excellence Commission. Model for Improvement and PDSA cycles Clinical Excellence Commission Website NSW Ministry of Health 2020 [Available from: http://www.cec.health.nsw.gov.au/Quality-Improvement-

Page $15 / 18$ 
Academy/quality-improvement-tools/model-for-improvement-and-pdsa-cycles.

20. Anderson KM, Wilson P, Odell PM, Kannel WB. An updated coronary risk profile. A statement for health professionals. Circulation. 1991;83(1):356-62.

21. May CR, Finch T, Ballini L, MacFarlane A, Mair F, Murray E, et al. Evaluating complex interventions and health technologies using normalization process theory: development of a simplified approach and web-enabled toolkit. BMC Health Services Research. 2011;11(1):245.

22. Dunham AH DJ, Johnson JK, Fuller J, Morgan M, Ford D. What attributions do Australian high-performing general practices make for their success? Applying the clinical microsystems framework: a qualitative study. BMJ Open. 2018;8:e020552.

23. Nieuwlaat R, Schwalm JD, Khatib R, Yusuf S. Why are we failing to implement effective therapies in cardiovascular disease? Eur Heart J. 2013;34(17):1262-+.

24. Welberry H, Barr ML, Comino EJ, Harris-Roxas BF, Harris E, Harris MF. Increasing use of general practice management and team care arrangements over time in New South Wales, Australia. Aust J Prim Health. 2019;25(2):168-75.

25. Chalasani S, Peiris DP, Usherwood T, Redfern J, Neal BC, Sullivan DR, et al. Reducing cardiovascular disease risk in diabetes: a randomised controlled trial of a quality improvement initiative. Med J Aust. 2017;206(10):436-41.

26. Glasgow RE, Tracy Orleans C, Wagner EH, Curry SJ, Solberg LI. Does the Chronic Care Model Serve Also as a Template for Improving Prevention? Milbank Quarterly. 2001;79(4):579-612.

27. Aggarwal M, Ornish D, Josephson R, Brown TM, Ostfeld RJ, Gordon N, et al. Closing Gaps in Lifestyle Adherence for Secondary Prevention of Coronary Heart Disease. Am J Cardiol. 2021;145:1-11.

28. Fuller J, Brown V, Ford D, Dunbar JA. The enablers and barriers for the uptake, use and spread of Primary Care Collaboratives in Australia. Australian Primary care conference; Adelaide: Australian National University; 2014.

29. Beaulieu-Jones BK, Lavage DR, Snyder JW, Moore JH, Pendergrass SA, Bauer CR. Characterizing and Managing Missing Structured Data in Electronic Health Records: Data Analysis. JMIR Med Inform. 2018;6(1):e11-e.

30. Bonner C, Jansen J, McKinn S, Irwig L, Doust J, Glasziou P, et al. General practitioners' use of different cardiovascular risk assessment strategies: a qualitative study. Med J Aust. 2013;199(7):485-9.

\section{Figures}




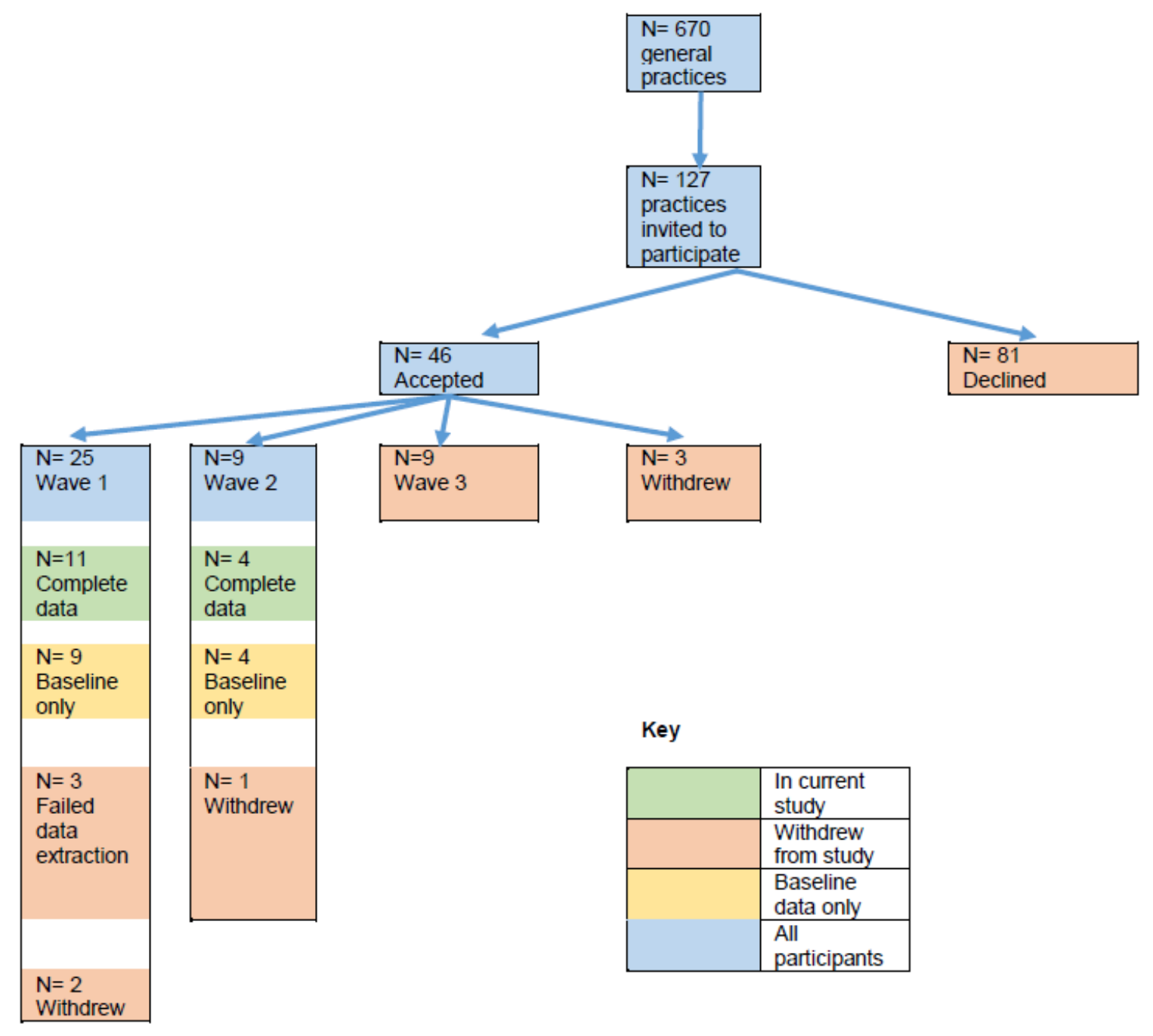

Figure 1

Practice Recruitment to the QPulse study 
1. Goal

Aim to increase the rate of measurement of waist circumference

2. Measure

Use measurement levels on PenCAT report pre and post intervention

3. Ideas

Talk to other Drs to remind them they need to do waist measurements

Check everyone has a tape measure in their rooms

Check everyone is measuring waist in the same way and recording it in the data space

P. Plan - Discuss at this weeks practice meeting/ ensure everyone aware we are going to report back in 1 months' time

D. Do- Practice nurse to do a weekly PenCAT report on waist circumference measurements recorded

S. Study - Report back at Doctors meeting by Nurse with results

A. Act - continue to report on a 6 monthly basis about Waist circumference measures with a goal to continuously increase our \% of those measured and recorded.

Outcome: Significant improvement in numbers with waist circumference measured from $0.7 \%$ pre-intervention to $18 \%$ post-intervention.

Figure 2

Plan-Do-Study-Act (PDSA) exemplar from one practice

\section{Supplementary Files}

This is a list of supplementary files associated with this preprint. Click to download.

- SupplementaryData.docx 